\title{
Adhesive Bowel Obstruction: Letter to the Editor
}

\section{Michael Cotton}

Published online: 5 February 2010

(c) Société Internationale de Chirurgie 2010

\section{Dear Sir,}

I read with interest the article by Butt et al. [1] regarding the rare but possible relevance of de novo adhesional bowel obstruction, i.e., cases of small bowel occlusion arising without previous abdominal surgery. In a study performed in Bulawayo, Zimbabwe [2], the pattern of small bowel obstruction changed with cases caused by postoperative adhesions becoming much more frequent than those due to inguinal hernia. However, a significant number of cases of small bowel obstruction in young women was found to be due to previous pelvic sepsis (PID) treated conservatively with antibiotics. This remains a considerable problem in resource-poor countries. An important impression was that de novo small bowel obstruction in men warranted early exploration because significant pathology was found in almost all cases. This message remains important in those circumstances where access to sophisticated scanning methods is unavailable.
Of course, it goes without saying that even more careful inspection of the abdomen is necessary in today's patient who may have had laparoscopic surgery with hardly any external scarring. Moreover, pervaginal or pergastric surgery leaves entirely no trace, thus forcing the examiner to rely on the patient's own recollection or records or those of his relatives.

\section{References}

1. Butt MU, Velmahos GC, Zacharias N et al (2009) Adhesional small bowel obstruction in the absence of previous operations: management and outcomes. World J Surg 33(11):2368-2371

2. Cotton MH (1995) Changing pattern of intestinal obstruction in Bulawayo, Zimbabwe. E Cent Afr J Surg 1:71-72
M. Cotton ( $\square)$

Médecin Associé aux Urgences, Centre Hospitalier Universitaire

Vaudois, 1011 Lausanne, Switzerland

e-mail: mikeytha@bluewin.ch 